\title{
Evaluation of neutrophil-to-lymphocyte ratio and hematologic parameters in patients with Graves' disease
}

\author{
Turan E \\ Department of Endocrinology and Metabolic Disease, Medical School of Bozok University, Yozgat, Turkey. \\ drelifturan@hotmail.com
}

\begin{abstract}
AIM: While the ratio of neutrophil-to-lymphocyte (NLR) increases with inflammation, its importance in Graves' disease is not clear. The aim of this study was to evaluate NLR, a marker of chronic inflammmation, in Graves' disease. METHODS: 86 Graves' patients (37 before treatment,49 euthyroid patients after treatment) and 112 controls were enrolled. Hematologic parameters, thyroid function tests, age and gender were recorded. NLRs were calculated. Firstly, groups were composed as Graves' group (Group1) and participants without thyroid disorder as control group (Group2). Secondly, Graves' patients before treatment were considered as Group1a, euthyroid Graves' patients after antithyroid treatment were considered as Group1b. These groups were compared with each other in terms of descriptive data and hematological parameters.

RESULTS: Lymphocyte, monocyte, platelet, free T3, and free T4 levels were significantly higher in Graves' group than the controls. TSH and NLR were significantly lower in Graves' group Graves' than the controls. Differences among group1a and group1b for monocyte $(p=0.013)$, for basophil $(p=0.002)$, for platelet $(p=0.029)$, and for NLR $(p=0.029)$ were statistically significant.

CONCLUSION: Unlike other inflammatory diseases, in Graves' disease; hematological parameters may not give information about inflammatory state of the disease. Therefore, NLR should be evaluated with other serum inflammatory markers in Graves' disease (Tab. 2, Fig. 1, Ref. 26). Text in PDF www.elis.sk. KEY WORDS: Graves' disease, inflammation, neutrophils, platelet count.
\end{abstract}

\section{Introduction}

Graves' disease is one of the autoimmune thyroid disorders and the most common reason of thyrotoxicosis. Thyrotoxicosis due to Graves' disease causes many changes in the whole body. Hematopoetic system is one of the altered systems. Blood cell levels can be affected by thyrotoxicosis (1). Besides thyrotoxicosis, antithyroid drugs may cause side effects in the hematological system (2-4).

Recently, neutrophil-to-lymphocyte ratio (NLR) as simple, non-invasive and cost-effective marker of inflammation, is frequently investigated in inflammatory diseases $(5,6)$ and malignancies (7).

The aim of this study was to evaluate NLR, a marker of chronic inflammmation, and hematological parametres in Graves' disease.

\section{Material and method}

In this study, over 18 years old, 86 (37 before treatment,49 euthyroid patients after treatment) participants with Graves' disease and age-sex matched 112 participants without thyroid dis-

Department of Endocrinology and Metabolic Disease, Medical School of Bozok University, Yozgat, Turkey.

Address for correspondence: E. Turan, Bozok University, Endocrinology, Adnan Menderes Street, 66040 Yozgat, Turkey.

Phone: +9003542127050

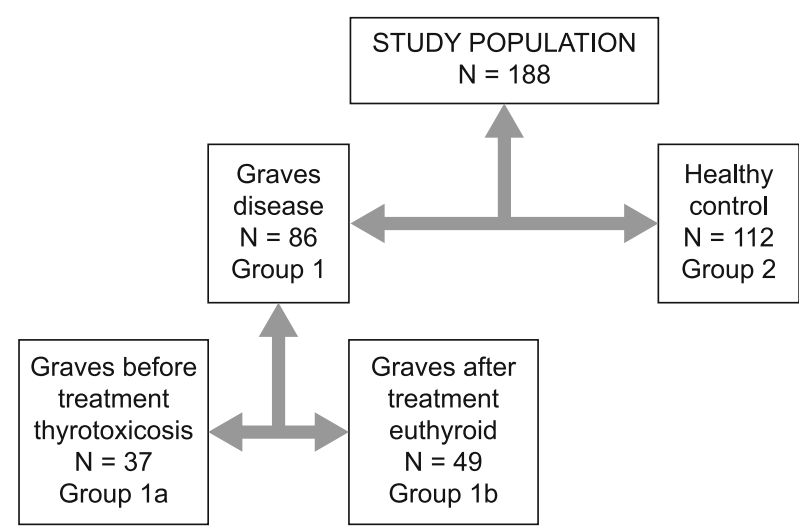

Randomization scheme

Fig. 1. Randomization scheme.

order were enrolled between January 2017 and September 2018 (Fig. 1). Graves' disease diagnostic criteria were: hyperthyroid symptoms, suppressed thyroid stimulating hormone levels (TSH normal range $0.35-4.95 \mathrm{mIU} / \mathrm{L}$ ), high free thyroxine (FT4 normal range $0.7-1.48 \mathrm{ng} / \mathrm{dL}$ ), high free triiodotropin (FT3 normal range $1.71-3.71 \mathrm{ng} / \mathrm{L}$ ), diffuse uptake in technetium scan and high TSH receptor antibody level (normal range 0-14 U/L). Analyses 
of free T3, free T4, TSH, anti-Tg, and anti-TPO were performed on DxI 800 Access Immunoassay (Beckman Coulter Inc., Brea, CA, USA) using a direct chemiluminescence detection system. TSH receptor antibody performed by electrochemiluminescence methods, using the system Cobas e801, Roche ${ }^{\circledR}$ Tc99m thyroid scintigraphy was performed with gamma camera interfaced dedicated computer system (Siemens E.CAM, Siemens Medical Systems Inc., Hoffman Estates, IL, USA). Thyroid ultrasonography was performed with an ultrasonography device (LOGIQ P6; GE Healthcare, Milwaukee, USA) equipped with a $10 \mathrm{MHz}$ linear probe. Hematological tests were measured using automated standard laboratory methods. Euthyroidism was defined as the levels of TSH, FT3 and FT4 in normal range. Methimazole was preferred as the first-line treatment for Graves' disease (8). Propylthiouracil was reserved for patients with side effects. Participants were categorized in two ways. Firstly; patients with Graves' disease were considered as Group 1, and the control subjects without thyroid disorder were considered as Group 2. These two groups were compared in terms of age, gender, leukocyte, neutrophil lymphocyte, monocyte, eosinophil, basophil, hemoglobin (hb), Free T3, Free T4, TSH levels, NLR, platelet to lymphocyte ratio (PLR). Secondly; patients with Graves' disease and overt hyperthyroidism, before treatment were considered as group 1a, euthyroid Graves' patients after 6 months of antithyroid treatment were considered as group $1 \mathrm{~b}$. These groups were compared among themselves. Exclusion criteria were; pregnancy, infection, rheumatologic and hematologic disorders.. The study was approved by the Bozok University ethics committee (Project no: 2017-KAEK189_2018.06.06_02). All procedures were in accordance with the ethical standards of the responsible committee on human studies (institutional and national) and with the Helsinki Declaration of 1975, as revised in 2008 .

All statistical analyses were calculated with the Statistical Package for the Social Sciences, version 18.0 (SPSS Inc., Chi-

Tab. 1. Descriptive features.

\begin{tabular}{lccc}
\hline & $\begin{array}{c}\text { Graves' disease } \\
(\mathrm{n}=86) \\
(\text { Group A) }\end{array}$ & $\begin{array}{c}\text { Control } \\
(\mathrm{n}=112)\end{array}$ & $\mathrm{p}$ \\
$($ Group B) & \\
\hline Age & $44.5 \pm 12.9$ & $43.1 \pm 12.7$ & 0.46 \\
Gender $(\mathrm{n}) \mathrm{male} /$ female & $61 / 25$ & $75 / 37$ & 0.59 \\
Leukocyte $10^{3} / \mu \mathrm{L}$ & $7.85 \pm 2.11$ & $7.64 \pm 2.29$ & 0.23 \\
Neutrophil $10^{3} / \mu \mathrm{L}$ & $4.42 \pm 1.51$ & $4.51 \pm 1.77$ & 0.78 \\
Lymphocyte $10^{3} / \mu \mathrm{L}$ & $2.63 \pm 1.03$ & $2.36 \pm 0.73$ & $\mathbf{0 . 0 4 7}$ \\
Monocyte $10^{3} / \mu \mathrm{L}$ & $0.61 \pm 0.2$ & $0.51 \pm 0.18$ & $\mathbf{0 . 0 0 3}$ \\
Eosinophil $10^{3} / \mu \mathrm{L}$ & $0.19 \pm 0.21$ & $0.19 \pm 0.23$ & 0.64 \\
Basophil $10^{3} / \mu \mathrm{L}$ & $0.04 \pm 0.028$ & $0.045 \pm 0.023$ & 0.757 \\
Hb g/dL & $13.9 \pm 1.67$ & $14.2 \pm 1.91$ & 0.25 \\
Platelet ng/dL & $288.31 \pm 66.19$ & $275 \pm 64.3$ & $\mathbf{0 . 0 3 9}$ \\
Free T3 ng/dL & $5.85 \pm 5.64$ & $2.76 \pm 0.33$ & $<\mathbf{0 . 0 0 1}$ \\
Free T4 ng/dL & $1.54 \pm 0.87$ & $1.01 \pm 0.26$ & $<\mathbf{0 . 0 0 0}$ \\
TSH mIU/L & $1.07 \pm 1.41$ & $1.88 \pm .01$ & $<\mathbf{0 . 0 0 1}$ \\
Neutrophil to lymphocyte ratio & $1.77 \pm 0.63$ & $2.11 \pm 1.21$ & 0.019 \\
Platelet to lymphocyte ratio & $118.1 \pm 38.67$ & $132.27 \pm 73.41$ & 0.62 \\
\hline Hb - Hemoglobin, TSH - Thyroid stimulating hormone, Free T3 - Free triiodo- \\
thyronine, FreeT4 - Free throxine, $\mathrm{p}$ - Pearson correlation Values are mean \pm SD.
\end{tabular}

Tab. 2. Values in Graves disease before and after treatment.

\begin{tabular}{lccl}
\hline & $\begin{array}{c}\text { Graves'disease } \\
\text { before treatment } \\
(\mathrm{n}=37)\end{array}$ & $\begin{array}{c}\text { Graves'disease } \\
\text { after treatment } \\
(\mathrm{n}=49)\end{array}$ & $\mathrm{p}$ \\
\hline Age & $43.40 \pm 13.2$ & $45.34 \pm 12.77$ & 0.6 \\
Gender $($ male/female $)$ & $9 / 28$ & $16 / 33$ & 0.6 \\
Leukocyte $10^{3} / \mu \mathrm{L}$ & $7.62 \pm 1.78$ & $7.98 \pm 2.34$ & 0.63 \\
Neutrophil $10^{3} / \mu \mathrm{L}$ & $4.13 \pm 1.25$ & $4.63 \pm 1.66$ & 0.35 \\
Lymphocyte $10^{3} / \mu \mathrm{L}$ & $2.65 \pm 0.62$ & $2.62 \pm 1.26$ & 0.09 \\
Monocyte $10^{3} / \mu \mathrm{L}$ & $0.68 \pm 0.22$ & $0.55 \pm 0.16$ & $<\mathbf{0 . 0 0 1}$ \\
Eosinophil $10^{3} / \mu \mathrm{L}$ & $0.16 \pm 0.13$ & $0.21 \pm 0.25$ & 0.58 \\
Basophil $10^{3} / \mu \mathrm{L}$ & $0.035 \pm 0.018$ & $0.054 \pm 0.031$ & 0.002 \\
Hb g/dl & $13.71 \pm 1.49$ & $14.08 \pm 1.80$ & 0.334 \\
Platelet $10^{3} / \mu \mathrm{L}$ & $306.02 \pm 64.03$ & $268.1 \pm 72.2$ & $\mathbf{0 . 0 2 9}$ \\
FreeT3 ng/dL & $9.94 \pm 6.69$ & $2.77 \pm 0.48$ & $<\mathbf{0 . 0 0 1}$ \\
FreeT4 ng/dL & $2.17 \pm 0.046$ & $1.07 \pm 0.46$ & $<\mathbf{0 . 0 0 1}$ \\
TSH mIU/L & $0.0039 \pm 0.0085$ & $1.88 \pm 1.41$ & $<\mathbf{0 . 0 0 1}$ \\
Neutrophil to lymphocyte ratio & $1.60 \pm 0.53$ & $1.89 \pm 0.67$ & $\mathbf{0 . 0 2 9}$ \\
Platelet to lymphocyte ratio & $119.35 \pm 32.05$ & $117.18 \pm 43.30$ & 0.26 \\
\hline
\end{tabular}

$\mathrm{Hb}$ - Hemoglobin, TSH - Thyroid stimulating hormone, Free T3 - Free triiodothyronine, FreeT4 - Free thyroxine, $\mathrm{p}-$ Pearson correlation. Values are mean \pm SD.

cago, IL, USA). P value $<0.05$ was considered as statistically significant. Baseline characteristics were expressed in numbers and percentage. Continuous variables were expressed as mean \pm standard deviation. To compare the two groups; $\chi^{2}$-test was used for categorical variables, student t-test was used for continuous variables with a normal distribution, and Mann-Whitney U-test for the others. In analysis of the three groups; One-way analysis of variance test was used to compare groups. Parameters with non-normal distribution were evaluated by using Kruskal Wallis.

\section{Results}

The characteristics of the participants for the two groups are shown in Table 1. In two groups analysis (Group 1 and 2); lymphocyte $(p=0.047)$, monocyte $(p=0.03)$, platelet $(p=0.039)$, free T3 $(p<0.001)$, and free T4 $(p<0.001)$ levels were significantly higher in Graves' disease group than in the control group. TSH $(\mathrm{p}<0.001)$ and NLR $(p=0.019)$ were significantly lower in Graves' group than the control group. PLR was similar among the groups $(p>0.05)$.

Group 1a and Group 1b analysis demonstrated significant difference between groups in terms of monocyte $(p=0.013)$, basophil $(p=0.002)$, platelet $(p=0.029)$ levels, NLR $(p=0.029))$, freeT4 $(p<0.001)$, free T3 $(p<0.001)$ and TSH levels $(p<0.001)$. Other parameters were similar between the groups (Tab. 2 ).

\section{Discussion}

This study showed that, incompatible with previous data on other autoimmune and inflammatory diseases, NLR was lower in Graves' disease with overt hyperthyroidism and after Graves' treatment a significant increase was observed.

Autoimmune diseases can be defined as disorders in which body's cells inexplicably attack its own tissues. Autoimmunity has the key role in Graves' disease pathogenesis. Due to the breach of immune tolerance, leukocytes and monocytes migrate to the thy- 
roid gland, causing progressive tissue damage. Neutrophils and lymphocytes play an important role in this process. Platelets are also responsible for pathogenesis. Platelets and lymphocytes act on each other's function. Platelets increase the adhesion and cell migration of helper T cells, cytolytic T cells, natural killer cells and B cells with the secretion molecules (9). Recently, white blood cells and subgroups (neutrophils, lymphocytes, monocytes, basophils, eosinophils) are easily detectable. These subgroups play a critical role in inflammation and cytokine release. Generally, neutrophilia and lymphocytopenia may be observed in tissue damage, stress and inflammation (10). Unlike other diseases with inflammation, Graves' disease may vary in white blood cell distribution, e.g, leucopenia, absolute and relative neutropenia, relative lymphocytosis (3). A previous study demonstrated that the prevalence of neutropenia in Graves' disease is $15-30 \%$ (11). Leucopenia may also be seen in hyperthyroid patients $(12,13)$. When we compared the group1 and 2, leukocyte and neutrophil counts were similar, and lymphocyte counts were significantly higher in Graves' patients compared to the control group. Van der Weerd K and et al reported that with flow cytometry method analysis, $\mathrm{T}$ and $\mathrm{B}$ lymphocyte counts were significantly higher in Graves' disease than in healthy control group and in the same study there was a positive correlation between the levels of $\mathrm{ft} 4$ and B lymphocytes (14).

Although antithyroid drugs are known to cause neutropenia as a side effect (15), Peng $Y$ and et al, reported that compared with untreated Graves' patients, neutrophil counts increase in Graves patients who are euthyroid with treatment (16). In this study neutrophil count increased but not statistically significantly. Klatka et al. reported that lymphocytes were slightly diminished with antithyroid treatment in Graves' disease (17). This finding can be connected with the anti-inflammatory effect of these drugs, because previous data suggest that methimazole may also decrease pathological inflammation (18).

Neutrophil-to-lymphocyte ratio and platelet-to-lymphocyte ratio are the recent indicators of severe inflammation. Several studies have investigated the relation of diabetes, hypertension, systemic diseases and malignancies with NLR $(19,20)$. Ulu et al. reported a significant association between NLR and diabetic retinopathy (21). Bilge et al. found that NLR and PLR were higher in euthyroid Hashimoto patients than healthy controls (22). Whereas, in a study by Ciray et al. there was no difference among NLR between the patients with or without diabetic retinopathy (23). Also there are studies in which Hashimoto thyroiditis, which is one of the autoimmune thyroid diseases, is found to be significantly higher than in the NLR and PLR control group (24) and before treatment than after levothroxine replacement (22). Despite the development of a similar autoimmune process with Graves' disease in Hashimoto thyroiditis, the thyroid hormone levels and the used medication are different. The effect on hematological parameters is known. In our study, in contrast to these results, PLR was similar between the groups and NLR was significantly lower in Graves' patients before treatment (Group1a) than euthyroid Graves' patients (Group1b). In accordance with previous data $(3,11)$, although not statistically significant, there was an increase in neutrophil and a decrease in lymphocyte count in Groupla, compared with group1b. As stat- ed earlier, unlike other inflammatory diseases, NLR levels were significantly lower in the Graves group and we can conclude that these results may probably be the result of increase in lymphocytes in Graves disease.

We found that monocyte and basophil counts were significantly higher in untreated Graves' patients than in euthyroid Graves' patients after anti-thyroid treatment and in the controls. Irvine et al. found that the monocyte count in Graves' patients was similar with the control group (3). There is not enough data about the basophil counts in Graves' disease.

Cases of thrombocytopenia due to hyperthyroidism have been reported in the literature (25). Although the mechanism is not clearly understood, the possible mechanism has been reported to be the immune-induced thrombocytopenia or hypersplenism in Graves' disease (26). On the other hand, in Graves' disease, megakaryocytes increase in bone marrow, while platelet count is normal, and platelet survival time is shortened (26). In our study, platelet counts were significantly increased in the Graves' group with overt hyperthyroidism compared to the controls and euthyroid Graves' patients.

There are some limitations of our study; firstly it was a retrospective study and we do not have an inflammation marker to compare our results, such as sedimentation, C-reactive protein, tumor necrosis factor, interleukin $1 \beta$, interleukin 6 . Secondly, we had a small number of patients in the groups. Thus, further large-scale studies are needed to prospectively evaluate these hematologic markers in Graves' disease.

\section{Conclusion}

Our results showed that NLR was decreased and platelet count was increased in untreated Graves' patients and these results were different from the literature. Significantly lower NLR in Graves' disease is thought to be compatible with the pathophysiology of the disease and antithyroid drug's suppressive effect on lymphocytes. NLR should be evaluated using other serum inflammatory markers in Graves' disease and larger prospective studies are needed to fully assess the benefits of NLR in patients with Graves'.

\section{References}

1. Melmed S SPK, Larsen P.R, Kronenberg HM. Williams Textbook of Endocrinology. United States of AMerica: Saunders Elsevier; 2011.

2. Cooper DS. Antithyroid drugs. New Engl J Med 2005; 352 (9): 905-917.

3. Irvine W, Wu F, Urbaniak S, Toolis F. Peripheral blood leucocytes in thyrotoxicosis (Graves' disease) as studied by conventional light microscopy. Clin Exp Immunol 1977; 27 (2): 216.

4. Sprikkelman A, Vellenga E. The application of hematopoietic growth factors in drug-induced agranulocytosis: a review of 70 cases. Leukemia 1994; 8 (12): 2031-2036.

5. Chittawar S, Dutta D, Qureshi Z, Surana V, Khandare S, Dubey TN. Neutrophil-lymphocyte ratio is a novel reliable predictor of nephropathy, retinopathy, and coronary artery disease in Indians with type- 2 diabetes. Indian J Endocrinol Metab 2017; 21 (6): 864. 
6. Kucuk A, Uslu AU, Ugan Y, Bagcaci S, Karahan AY, Akarmut A et al. Neutrophil-to-lymphocyte ratio is involved in the severity of ankylosing spondylitis. Bratisl Med J 2015; 116 (12): 722-725.

7. Ramos-Esquivel A, Rodriguez-Porras L, Porras J. Neutrophil-lymphocyte ratio and platelet-lymphocyte ratio as prognostic factors in nonmetastatic breast cancer patients from a Hispanic population. Breast Dis 2017; 37 (1): 1-6.

8. Kahaly GJ, Bartalena L, Hegedüs L, Leenhardt L, Poppe K, Pearce SH. 2018 European Thyroid Association Guideline for the Management of Graves' Hyperthyroidism. Eur Thyroid J 2018; 7 (4): 1-20.

9. Li N. Platelet-lymphocyte cross-talk. J Leukocyt Biol 2008; 83 (5): 1069-1078.

10. Zahorec R. Ratio of neutrophil to lymphocyte counts-rapid and simple parameter of systemic inflammation and stress in critically ill. Bratisl Med J 2001; 102 (1): 5-14.

11. Eakin DL, Peake RL, Weiss GB. Effect of therapy on the neutropenia of hyperthyroidism. Southern Med J 1983; 76 (3): 335-337.

12. Talansky AL, Schulman P, Vinciguerra VP, Margouleff D, Budman DR, Degnan TJ. Pancytopenia complicating Graves' disease and drug-induced hypothyroidism. ArchIntern Med 1981; 141 (4): 544-545.

13. Kinjo Y. A case of Graves' disease with myelodysplastic syndrome, in which Mercazole improved pancytopenia. Clin Endcrinol 2000; 48: 60-63.

14. Van der Weerd K, Van Hagen P, Schrijver B, Kwekkeboom DJ, De Herder W, Ten Broek M et al. The peripheral blood compartment in patients with $\mathrm{G}$ raves' disease: activated $\mathrm{T}$ lymphocytes and increased transitional and pre-naive mature B lymphocytes. Clin Exp Immunol 2013; 174 (2): 256-264.

15. Süreli TMGÇU, Kullanımına P, Nötropeni B. Neutropenia Due to Very Long Time Propylthiouracil Usage in Toxic Multinodular Goiter. 2016.

16. Peng Y, Qi Y, Huang F, Chen X, Zhou Y, Ye L et al. Down-regulated resistin level in consequence of decreased neutrophil counts in untreated Grave's disease. Oncotarget 2016; 7 (48): 78680-78687.
17. Klatka M, Grywalska E, Surdacka A, Tarach J, Klatka J, Rolinski J. Peripheral blood lymphocyte apoptosis and its relationship with thyroid function tests in adolescents with hyperthyroidism due to Graves' disease. Arch Med Sci 2012; 8 (5): 865-873.

18. Wenisch C, Myskiw D, Gessl A, Graninger W. Circulating selectins, intercellular adhesion molecule-1, and vascular cell adhesion molecule-1 in hyperthyroidism. J Clin Endocrinol Metab 1995; 80 (7): 2122-2126.

19. Faria SS, Fernandes Jr PC, Silva MJB, Lima VC, Fontes W, Freitas-Junior $\mathbf{R}$ et al. The neutrophil-to-lymphocyte ratio: a narrative review. Ecancermedicalscience 2016; 10.

20. Guthrie GJ, Charles KA, Roxburgh CS, Horgan PG, McMillan DC, Clarke SJ. The systemic inflammation-based neutrophil-lymphocyte ratio: experience in patients with cancer. Crit Rev Oncol Hematol 2013; 88 (1): 218-230.

21. Ulu SM, Dogan M, Ahsen A, Altug A, Demir K, Acartürk G et al. Neutrophil-to-lymphocyte ratio as a quick and reliable predictive marker to diagnose the severity of diabetic retinopathy. Diabet Technol Ther 2013; 15 (11): 942-947.

22. Bilge M, Yesilova A, Adas M, Helvaci A. Neutrophil- and Plateletto Lymphocyte Ratio in Patients with Euthyroid Hashimoto's Thyroiditis. Exp Clin Endocrinol Diabet 2018; 126.

23. Ciray H, Aksoy AH, Ulu N, Cizmecioglu A, Gaipov A, Solak Y. Nephropathy, but not Angiographically Proven Retinopathy, is Associated with Neutrophil to Lymphocyte Ratio in Patients with Type 2 Diabetes. Exp Clin Endocrinol Diabet 2015; 123 (5): 267-271.

24. Aktas G, Sit M, Dikbas O, Erkol H, Altinordu R, Erkus E et al. Elevated neutrophil-to-lymphocyte ratio in the diagnosis of Hashimoto's thyroiditis. Revista da Associacao Med Brasil 2017; 63 (12): 1065-1068.

25. Adrouny A, Sandler RM, Carmel R. Variable presentation of thrombocytopenia in Graves' disease. Arch Intern Med 1982; 142 (8): 1460-1464.

26. Panzer S, Haubenstock A, Minar E. Platelets in hyperthyiroidism: studies on platelet counts, mean platelet volume, 111-indium-labeled platelet kinetics, and platelet-associated immunoglobulins G and M. J Clin EndocrinolMetab 1990; 70 (2): 491-496. 